\title{
Vagus Nerve Stimulation
}

\author{
Robert H. Howland
}

Published online: 7 March 2014

(C) Springer International Publishing AG 2014

\section{Introduction}

The sympathetic and parasympathetic components of the autonomic nervous system (ANS) control and regulate the function of various organs, glands, and involuntary muscles throughout the body (e.g., vocalization, swallowing, heart rate, respiration, gastric secretion, and intestinal motility). The vagus nerve (cranial nerve $\mathrm{X}$ ) is a mixed nerve composed of $20 \%$ "efferent" fibers (sending signals from the brain to the body) and $80 \%$ "afferent" (sensory) fibers (carrying information from the body to the brain). The efferent cholinergic fibers are the main parasympathetic component of the ANS $[1 \bullet \cdot]$, but an important function of the vagus nerve is transmitting and/or mediating sensory information from throughout the body to the brain [2]. The right and left vagus nerves exit from the brainstem, and they course through the neck (in the carotid sheath between the carotid artery and jugular vein), upper chest (along the trachea), lower chest and diaphragm (along the esophagus), and into the abdominal cavity [3•]. During this course, branches enervate various structures such as the larynx, pharynx, heart, lungs, and gastrointestinal tract. In the brainstem, the sensory afferent fibers terminate in the nucleus tractus solitarius, which then sends fibers that connect directly or indirectly to different brain regions. These regions include the dorsal raphe nuclei, locus ceruleus, amygdala, hypothalamus, thalamus, and orbitofrontal cortex.

The term "vagus nerve stimulation" (VNS) can be used generally to describe any technique that stimulates the vagus nerve. An observation first made in the 1880 s was that manual massage and compression of the carotid artery in the cervical region of the neck could suppress seizures, an effect attributable to crude stimulation of the vagus [4]. Electrical VNS studies were conducted during the 1930s and 1940s to understand the influence of the ANS on modulating brain activity. Studies in cats and monkeys demonstrated that VNS influenced brain electrical activity. Subsequent studies determined 
that VNS had anticonvulsant effects on experimentally induced seizures in dogs [5]. Various forms of paced breathing can also influence brain electrical activity, which might be mediated by VNS arising from the diaphragm [6, 7]. Cardiorespiratory stimulation of the vagus nerve may explain some of the positive emotional and cognitive benefits of deep breathing, yoga, or aerobic exercise activities. Dedicated clinical trials eventually led to approval by the US Food and Drug Administration (FDA) of an implanted VNS device indicated for the treatment of refractory epilepsy in 1997 [8•]. The same device was later given an FDA-approved indication for the treatment of chronic treatment-resistant depression (chronic TRD) in 2005. Small open-label studies and case series reports have described the use of VNS for rapid cycling bipolar disorder, treatment-resistant anxiety disorders, Alzheimer disease, chronic refractory headaches, and obesity, although none of these uses has been given FDA approval [9].

\section{Methods of Vagus Nerve Stimulation (VNS)}

\section{Left Cervical VNS}

The most common clinical use of VNS involves the surgical implantation of a commercially available programmable pulse generator device (NCP System; Cyberonics, Inc., Houston, TX, USA) [10]. The implant surgery is performed under general anesthesia, typically as an outpatient procedure. The generator is implanted subcutaneously in the left upper chest or left axillary border. The electrode lead wire is attached to the left mid-cervical vagus nerve through a second incision in the left neck area. The lead wire is passed through a subcutaneous tunnel and attached to the pulse generator. Possible surgical complications include wound infection and hoarseness (due to temporary or permanent left vocal cord paralysis), which occurs in about $1 \%$ of patients.

A handheld computer programs the pulse generator stimulation parameters via a programming wand placed on the skin over the device. The programmable parameters are the current charge [electrical stimulus intensity, measured in milliamperes $(\mathrm{mA})]$, the pulse width (electrical pulse duration, measured in microseconds), the pulse frequency [measured in Hertz (Hz)], and the on/off duty cycle (the stimulus on-time and off-time, measured in seconds or minutes). Initial settings for the four parameters can each be adjusted to optimize efficacy (for seizure control or for other symptom control depending on the indication) and tolerability. The generator runs continuously, but patients can turn off VNS temporarily by holding a magnet over the device and VNS can be turned on and off by the programmer. The pulse generator battery life depends on the stimulus parameters, and it can be replaced or permanently removed in a simple surgical procedure.
The adverse effects of VNS are mostly stimulation related and therefore experienced for very short intermittent periods of time. Possible adverse effects could be related to the stimulation of any body structure enervated by the vagus nerve, but $80 \%$ of fibers are afferent and these electrical pulses are propagated from the point of attachment toward the brain rather than the body. Stimulation from the left mid-cervical vagus nerve most commonly causes voice alteration, cough, dyspnea, dysphagia, and neck pain or paresthesias. Left cervical VNS is believed to minimize potential cardiac effects such as bradycardia or asystole (primarily mediated by the right vagus nerve). Stimulation parameters can be adjusted to make adverse effects more tolerable, but tolerance often occurs with chronic stimulation. Experience in epilepsy populations has shown that VNS is effective, safe, and well tolerated in pediatric patients. There are no identified risks when VNS has been used during pregnancy. VNS is safe and compatible to use together with psychotropic drugs and with electroconvulsive therapy (ECT). Whole body magnetic resonance imaging (MRI) scans cannot be done with VNS implants, but MRI scans of the head are possible using a transmit/receive head coil [11]. Shortwave, microwave, or therapeutic ultrasound diathermy should not be used, but diagnostic ultrasound is safe. Metal detectors, microwave ovens, cellular telephones, and other electrical or electronic devices will not affect VNS.

\section{Right Cervical VNS}

Right cervical VNS reduces seizure activity in animal models, and there is some evidence that this is true in humans [3•], but it is not known whether right cervical VNS would effectively treat depressive symptoms. A VNS device system (CardioFit System; BioControl Medical Ltd, Yehud, Israel) has been

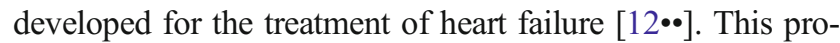
grammable device is implanted in the right chest wall. It is connected to the right cervical vagus using a cuff designed to preferentially activate vagal efferent fibers (intended to affect cardiac function). The stimulator senses heart rate and shuts off at a predetermined threshold of bradycardia. Preclinical studies and one phase II human study suggest that chronic right cervical VNS is safe and effective for treating heart failure [12••, 13••]. A similar VNS system (FitNeS System; BioControl Medical Ltd) has been designed with a cuff electrode that preferentially activates afferent fibers, which is intended to minimize typical VNS side effects related to efferent fiber stimulation. Left cervical VNS using this device has been described in five patients with epilepsy, who showed some benefit and no typical VNS side effects [14].

\section{Transcutaneous Forms of VNS}

The outer ear is supplied by three sensory nerves: the auriculotemporal nerve, the great auricular nerve, and the 
auricular branch of the vagus nerve $(\mathrm{ABVN})[15 \bullet \cdot]$. The external auditory meatus and concha (cymba conchae and cavum conchae) of the ear are supplied mainly by the $\mathrm{ABVN}$, and the cymba conchae is supplied exclusively by the ABVN. A transcutaneous method of VNS (t-VNS) targets the cutaneous receptive field of the ABVN. Applying an electrical stimulus to the left cymba conchae (using a stimulus intensity above the sensory detection threshold, but below the pain threshold) results in a brain activation pattern not dissimilar to that of left cervical VNS $[16,17,18 \bullet \cdot$. The use of t-VNS for treating epilepsy was first proposed in 2000 [19]. A t-VNS device (NEMOS; Cerbomed GmbH, Erlangen, Germany) received European clearance for the treatment of epilepsy and depression in 2010 and for the treatment of pain in 2012. These approvals were based primarily on preclinical studies of t-VNS as well as extrapolating the findings from preclinical and human studies of left cervical VNS.

Transcutaneous electrical nerve stimulator (TENS) devices can also be employed to administer t-VNS, by situating contact electrodes in the region of the cymba conchae. Patients can self-administer t-VNS, which can be applied unilaterally or bilaterally (depending on the device system used), but there is no established clinical paradigm for how t-VNS should be administered (i.e., stimulation parameters; duration and frequency of each stimulation session; length of treatment). The NEMOS manufacturer suggests that each session should last at least $1 \mathrm{~h}$ and should be used three to four times per day, but the basis for this recommendation is unclear. There is some published clinical data (mostly pilot studies) on the use of t-VNS for epilepsy, depression, pain, and other clinical indications, suggesting that it is safe and well tolerated $[20,21,22 \bullet \bullet, 23 \bullet, 24,25 \bullet \bullet]$.

Another type of t-VNS device (gammaCore; electroCore LLC, Basking Ridge, NJ, USA) has European clearance for the prophylactic and acute treatment of cluster headache, migraine, hemicrania continua, and medication overuse headache. Therapy using gammaCore is delivered through a hand-held portable device with two flat stimulation contact surfaces that transmits a proprietary electrical signal in the vicinity of the vagus nerve. The device is placed on the neck over the vagus nerve, at a location where the pulse is found. The stimulation intensity is controlled by the patient and the application stimulation lasts for $90 \mathrm{~s}$. Patients may experience headache relief when used as needed, but the device can be used several times per day to prevent headaches. Pilot studies and case series reports using the gammaCore device for primary headache syndromes have been described in abstract form and larger controlled trials are underway [26], but this device has not been investigated in epilepsy or depression.

\section{Studies of VNS for Depression}

\section{Rationale for VNS in Depression}

The rationale for investigating VNS as a treatment for depression is based on various preclinical and clinical studies [27]. In animal models of depression, VNS has antidepressant-like effects $[28,29]$. VNS has positive effects on mood symptoms in epilepsy, even among those patients whose seizures do not improve. The vagus nerve has direct and indirect connections to the cortical-limbic-thalamic-striatal neural circuit pertinent to emotional and cognitive functions relevant in depression $[1 \bullet \cdot]$. Functional brain imaging studies in humans demonstrate that VNS influences physiological activity in these areas [30•, 31•]. Animal and human studies have shown that VNS influences the activity of norepinephrine, serotonin, and other neurotransmitters implicated in mood disorders $[1 \bullet \cdot]$. Like other antidepressant therapies, VNS increases the expression of the neurotrophin brain-derived neurotrophic factor and activates its receptor [32], and also stimulates hippocampal neurogenesis [33].

\section{Clinical Trials of Left Cervical VNS for Depression}

An open-label pilot study first investigated left cervical VNS in 60 patients with chronic TRD [34, 35]. These patients had unipolar or bipolar depression, were depressed for an average of nearly 10 years, and had not responded to an average of 16 different antidepressant therapies. After implant, 2 weeks of post-surgery recovery without stimulation was followed by 10 weeks of active VNS. Approximately $30 \%$ responded and $15 \%$ achieved remission. Patients who had not responded previously to ECT were less likely to respond. Among 13 patients who had not responded to more than seven different drug treatments previously, none responded to VNS. Of the remaining patients (fewer than seven prior treatment failures), $39 \%$ responded to VNS. During follow-up of 59 patients, $44 \%$ were responders ( $27 \%$ remitters) at 1 year [36] and $42 \%$ were responders $(22 \%$ remitters $)$ at 2 years [37]. These outcomes demonstrated that the effectiveness of VNS increased with time and was maintained, which is contrary to typical experience with pharmacotherapy in chronic TRD [38]. Patients with fewer previous unsuccessful treatments were more likely to respond or remit during long-term VNS therapy. By 2 years, two patients had died (unrelated to VNS), four had dropped out, and 48 (81 \%) were still receiving VNS. The $81 \%$ retention rate suggested that VNS was an acceptable treatment and that patients may have derived non-specific therapeutic benefits from the treatment even if they had not achieved a response or remission based on depression rating scale assessments.

A multi-center, randomized, double-blind, controlled, acute treatment study comparing active (device turned on) 
with sham (device turned off) left cervical VNS in 235 patients with chronic TRD was subsequently conducted [39]. These patients had unipolar or bipolar disorder, and had not responded to between two and six different antidepressant therapies. After implant, 2 weeks of post-surgery recovery without stimulation was followed by 10 weeks of active or sham VNS. Response rates (15\% active vs. $10 \%$ sham) based on the primary efficacy outcome measure [Hamilton Rating Scale for Depression (HRSD)] were not significantly different, but there was a significant difference in response rates ( $17 \%$ active vs. $7 \%$ sham) based on a secondary measure, the Inventory of Depressive Symptomatology-Self Report. Only three patients $(1 \%)$ dropped out because of adverse events.

At the end of the acute study, VNS was activated in all patients and 205 were followed in a long-term naturalistic treatment study [40]. A cohort of 124 patients having chronic TRD and receiving treatment as usual (TAU) was enrolled as a naturalistic comparison group [41]. After 1 year, VNS patients were significantly more likely to be improved ( $27 \%$ response; $16 \%$ remission) compared with TAU patients (13\% response; $7 \%$ remission). This study confirmed that the antidepressant response to VNS tends to increase over time and the majority of patients maintain their response. Only $3 \%$ of VNS patients dropped out because of adverse events; $90 \%$ continued treatment. There was no difference in treatment outcomes for patients with bipolar versus unipolar depression [42].

Schlaepfer and colleagues [43] reported the results of an open-label, uncontrolled, European, multicenter study of left cervical VNS in 74 patients with chronic unipolar or bipolar TRD (non-response to two to six antidepressant therapies). Half the patients had previously received ECT, including $38 \%$ who had received ECT in their current episode. During the first 3 months post-implant, medication doses and VNS stimulation parameters were kept stable. For the next 9 months, medications and VNS stimulation parameters were changed if necessary. After 3 months, the response rate (based on the HRSD) was $37 \%$ and the remission rate was $17 \%$. The 1 - and 2 -year response rates were $53 \%$ and $53 \%$, respectively, and the respective remission rates were $33 \%$ and $40 \%$ [44]. Two patients exited the study because of adverse events, two had VNS explanted, and two committed suicide.

\section{Dosing Studies of VNS for Depression}

The safety and effectiveness of three different stimulation levels of left cervical VNS were investigated in a randomized, double-blind, multicenter study of 331 patients with unipolar or bipolar TRD (non-response to four or more previous antidepressant therapies) [45•]. Fifty-seven percent of the patients had previously received ECT, $81 \%$ had not responded to six or more antidepressants in their current depressive episode, and nearly half had previously attempted suicide. Patients were randomized to one of three VNS dose-parameter groups: Low $(0.25 \mathrm{~mA}$ current; $130 \mathrm{~ms}$ pulse width), Medium $(0.5-1.0 \mathrm{~mA} ; 250 \mathrm{~ms})$, or High (1.25-1.5 mA; $250 \mathrm{~ms})$. Stimulation parameters were stable within each group for the first 22 weeks (acute phase), after which the output current could be increased if necessary until week 50 (end of study). During the acute phase, all groups showed statistically significant improvement on the primary efficacy measure (change from baseline on the Inventory of Depressive Symptomatology-Clinician Rated scale), but there were no differences between groups. Mean change in these scores showed continued improvement by week 50. A composite measure of total charge delivered per day was significantly correlated with depressive symptom improvement (i.e., a greater total charge was associated with decreasing depressive symptoms). Response and remission rates (for each group) were numerically higher at week 50 than at week 22 , but there were no significant differences of response or remission rates among the groups at week 22 or at week 50 . The proportion of responders at week 22 who were also responders at week 50 was substantially higher for the Medium and High groups than for the Low group. Six patients died (two by suicide; four unrelated to the study); $94 \%$ remained in the study until week 50 .

The antidepressant effects of two different VNS stimulation parameters were also evaluated by Muller and colleagues [46] in a retrospective analysis of data derived from two parallel groups of ten patients: Low strength/high frequency $(\leq 1.5 \mathrm{~mA} ; 20 \mathrm{~Hz}) \mathrm{vs}$. high strength/low frequency $(>1.5 \mathrm{~mA}$; $15 \mathrm{~Hz}$ ). At follow-up (duration of VNS treatment was not specified), there was a significant decrease in the HRDS for the low-strength/high-frequency group, but not the other group.

\section{Meta-analysis of VNS Studies for Depression}

Berry and colleagues [47••] recently published a metaanalysis of patient-level data from six multicenter studies of left cervical VNS for TRD. This analysis included patient data from five of the published studies reviewed above and data from an ongoing nonrandomized registry study [48•]. The registry compares VNS plus TAU (VNS+TAU; 335 patients) with TAU (301 patients). The objective of the meta-analysis was to compare response and remission rates [based on the Montgomery-Åsberg Depression Rating Scale (MADRS) and the Clinical Global Impressions Improvement (CGI-I) subscale] over time for VNS+TAU (1035 subjects) and TAU (425 subjects) from patient-level data, rather than treatment effect sizes, derived from the six studies. The MADRS response rate for VNS+TAU at $12,24,48$, and 96 weeks was $12 \%, 18 \%$, $28 \%$, and $32 \%$ vs. $4 \%, 7 \%, 12 \%$, and $14 \%$ for TAU. The MADRS remission rates were $3 \%, 5 \%, 10 \%$, and $14 \%$ for 
VNS+TAU vs. $1 \%, 1 \%, 2 \%$, and $4 \%$ for TAU. When data were analyzed by odds ratios, VNS+TAU was associated with a significantly greater likelihood of response and remission compared with TAU. For patients who had responded to VNS+TAU at 24 weeks, sustained response was more likely at 48 weeks and at 96 weeks. The analysis of response and remission rates based on the CGI-I similarly distinguished the VNS+TAU and TAU groups.

\section{Safety and Tolerability of VNS for Depression}

The relative safety, tolerability, and acceptability of VNS in the depression studies are comparable to those seen in epilepsy [8•]. The most common adverse effects were voice alteration, cough, dyspnea, dysphagia, and neck pain or paresthesias; were typically mild-moderate in severity; and often improved with time or by adjusting VNS. Insomnia, sedation, sexual dysfunction, weight gain, and cognitive impairment were not associated with VNS. The risk of developing hypomania or mania is low [49॰]. VNS was not associated with an increased risk of suicidal thoughts, suicide attempts, or completed suicide in the depression studies. Depression-related suicidal symptoms often improved over time.

Additional data from the VNS registry found that VNStreated patients had lower rates of all-cause mortality, completed suicide, suicide thoughts, and suicide attempts compared with TAU patients, although not all of the findings were statistically significant [48•]. In a retrospective analysis of Medicare administrative claims data, Feldman and colleagues [50] compared the experience of four groups of Medicare beneficiaries: those receiving left cervical VNS; those defined as having TRD; those defined as having non-TRD; and those defined as general Medicare beneficiaries. Patients receiving VNS had lower annual mortality rates compared with the three other groups. The medical costs per patient-year were found to be similar for the VNS and non-TRD groups, both of which were substantially lower than for the TRD group.

\section{Transcutaneous VNS for Depression}

As described previously, auricular t-VNS is an alternative form of stimulating the vagus nerve. Hein and colleagues [25*0] conducted a randomized, sham-controlled, pilot study of auricular t-VNS in 37 patients with major depression (not TRD). The t-VNS was administered bilaterally using a TENS microstimulator unit (manufactured by Auri-Stim Medical Inc., Denver, CO, USA). The stimulation was used for 15 min once or twice a day, 5 days per week, for 2 weeks. Active stimulation was set just below the threshold of perception; sham stimulation involved no current. Active stimulation was associated with a significantly greater improvement on a self-report measure of depression (the Beck Depression Inventory) compared with sham stimulation after 2 weeks, but there was no change within or difference between groups on the HRSD. The treatment was well tolerated. Another study of auricular t-VNS for non-chronic depression is currently being conducted [51].

\section{Depression, Inflammation, Metabolic Syndrome, Infectious Disease, Heart Disease, and VNS}

Inflammatory Biomarkers, Depression, and the Heart

Studies have shown that depression is an independent risk factor for the development of cardiovascular disease, and that comorbid depression increases the morbidity and mortality of patients with pre-existing heart disease [52, 53]. Depression also contributes to the risk of developing cardiac arrhythmias [54]. Heart failure resulting from cardiac ischemia or tachycardia is accompanied by changes in autonomic tone resulting in increased heart rate and decreased heart rate variability. Autonomic dysfunction in heart failure is often associated with neurohormonal activation (e.g., increased plasma norepinephrine, angiotensin II, and endothelin-1), inflammatory biomarkers and cytokines (e.g., tumor necrosis factor alpha and C-reactive protein), metabolic changes, and increased systemic and cardiac oxidant stress [55]. Similarly, autonomic dysregulation, inflammatory/immune system activation, and dysregulated neuro-immune system interactions are implicated in hypertension and cardiovascular disease [56]. There is extensive experience using measures of heart rate variability in diverse disease syndromes, and these studies indicate that decreased heart rate variability and decreased vagus nerve activity is associated with increased morbidity and mortality [57].

Complex bidirectional relationships among depression, immune system function, and infectious disease have been described $[58,59]$. There is considerable evidence from preclinical and clinical studies that acute or chronic infectious disease can increase the risk of developing depression, and this causal effect is mediated by the induction of proinflammatory cytokines [60-62]. Cytokine receptors have been identified in the brain, and cytokines have been shown to activate the hypothalamic-pituitary-adrenal (HPA) axis and to alter neurotransmitter function, both of which are relevant to the pathogenesis of depression. Gut microbiota has a role in priming and regulating whole-body immunoregulatory activity and can influence brain function and behavior [63, 64]. The state of depression itself does not cause elevated levels of inflammatory cytokines [65].

The Vagus Nerve and the Neuro-endocrine-immune Axis

The vagus nerve is a major component of the ANS, has an important role in the regulation of metabolic homeostasis, and 
plays a key role in the neuro-endocrine-immune axis to maintain homeostasis through its afferent and efferent pathways $[66 \bullet \bullet, 67 \bullet \bullet]$. Stimulation of receptors expressed on immune cells by pathogen-associated molecular patterns or diseaseassociated molecular patterns induces an increase of proinflammatory cytokines that communicate with the brain through neural and humoral pathways. The vagus nerve mediates an anti-inflammatory effect through its afferent pathways involved in the activation/regulation of the HPA axis and adrenal gland corticosteroid release. By contrast, vagus nerve efferents mediate anti-inflammatory processes via direct effects on immune cells or through the splenic sympathetic nerve. This pathway is referred to as the cholinergic antiinflammatory pathway (CAP). Activation of vagal afferents by inflammatory mediators (such as cytokines) in peripheral tissues results in an inflammatory reflex in which vagal efferents inhibit inflammation by suppressing cytokine production via the CAP.

Experimental studies (e.g., animal models of endotoxemia, sepsis, shock, and colitis) have demonstrated that invasive VNS significantly alters proinflammatory cytokines and other measures of inflammation. Right and left VNS also has favorable effects on focal cerebral ischemia in animal studies and this does not appear to be related to alterations in cerebral blood flow [68, 69]. Transcutaneous VNS was shown to reduce proinflammatory biomarkers and improve survival in a murine sepsis model [70]. Clinical studies of inflammatory markers in VNS-treated epilepsy patients are limited. Some of these studies, but not all, have demonstrated alterations associated with VNS [71-74]. It should be noted that these patients had epilepsy and were not studied under conditions of inflammation. Corcoran and colleagues [75] measured various peripheral cytokines before and 3 months after left cervical VNS implantation in ten patients with TRD. They found increases in certain proinflammatory and anti-inflammatory peripheral cytokines.

Levels of inflammatory cytokines and nitric oxide synthase [NOS; an enzyme that catalyzes nitric oxide (NO), which is a highly reactive free radical oxygen species that functions as a gaseous signaling molecule] are elevated in heart failure. In experimental animal studies of heart failure, VNS modulates the inflammatory response and NOS production [55]. In humans, stress and depression have been associated with changes in NO [76, 77]. Interferon-alfa has been shown to induce NOS and NO release [78]. Interferon-alfa is an innate immune cytokine that has anti-viral and anti-proliferative activities, is a potent stimulator of proinflammatory cytokines in the periphery and central nervous system, and induces depression. Whether VNS influences NOS or NO in humans with heart disease or depression is unknown.

Various antidepressant therapies can suppress or attenuate proinflammatory processes [79••], although the presence of inflammatory biomarkers is associated with a less than optimal response to standard antidepressant drugs [80, 81]. As a result, novel pharmacologic agents that target inflammatory processes, either directly or indirectly, are of interest as potential antidepressant therapies and are now being investigated $[80,81]$. The central role of the vagus nerve in the regulation of the neuro-endocrine-immune axis might be a potential mechanism of action for VNS in the treatment of depression. Moreover, the known associations between depression, cardiovascular disease, cerebrovascular disease, metabolic syndrome, infectious disease, and other health conditions suggest that using VNS as a treatment for depression might have primary, secondary, and/or tertiary prevention benefits for many or all of these associated conditions [67••, 82]. If so, overall morbidity and mortality might be favorably influenced by long-term VNS therapy and might be safer than antidepressant drugs [83].

\section{Conclusions}

\section{The Relative Antidepressant Effectiveness of VNS}

Left cervical VNS is an approved therapy for epilepsy and for TRD, but its antidepressant efficacy is modest. In a critical review, Martin and Martin-Sanchez [84] concluded that insufficient data are available to even describe VNS as effective in the treatment of depression. They suggest that its positive effects may be mediated by the placebo effect, regression toward the mean, spontaneous remission, or the Hawthorne effect; that it is not clear that its potential benefit is outweighed by possible harm; and that solid evidence of an antidepressant effect should be based exclusively on long-term clinical trials with a control group.

In their analysis, however, the authors mix epilepsy studies and depression studies. They also fail to discuss the unique nature of the patient population enrolled in the VNS depression studies [85]. These depressed patients are distinguished by the chronicity of their depressive disorder (average length of illness more than 25 years and average length of current episode nearly 7 years), their extensive history of treatment non-response (average of seven drug failures and more than $50 \%$ having received ECT), and their rates of lifetime hospitalizations and suicide attempts. Such patients are invariably excluded from most clinical treatment trials, except perhaps for studies of deep brain stimulation [9]. A placebo effect, spontaneous remission, or simply observing these patients (the Hawthorne effect) are unlikely to explain even modest improvements (compared with TAU) among patients with these clinical characteristics. The tolerability and safety of implanted VNS systems have been well established based on worldwide experience with epilepsy and depression patients, including children and adolescents [8•]. Conducting a 
long-term controlled trial in TRD would be scientifically ideal, but technically challenging and ethically problematic.

In the Sequenced Treatment Alternatives to Relieve Depression (STAR*D) trial [86], the majority of patients had chronic or recurrent depression, but they were not considered treatment resistant and they were less severely and chronically ill than patients enrolled in the VNS studies. Treatment was rendered sequentially through four levels (each lasting up to 12 weeks) of various antidepressant therapies. Patients with an unsatisfactory treatment response at any level moved to the next level. Rates of intolerance, defined as the proportion of patients exiting a level because of adverse events, were in the range of $16-35 \%$ at each of the four levels. Altogether, the cumulative remission rate after up to four levels of treatment was $67 \%$. About $33 \%$ of patients therefore did not achieve remission after up to four levels of treatment, and a substantial minority was treatment intolerant. Relapse was assessed during a 1-year naturalistic follow-up of patients who had a satisfactory treatment response. Patients who required more treatment steps had higher relapse rates during the naturalistic follow-up phase: $40 \%, 55 \%, 65 \%$, and $71 \%$ after each of the four sequential levels, respectively. At any level, lower relapse rates were found among patients who were in remission at follow-up entry than for those who were not in remission. The efficacy, tolerability, and relapse rates after 1 year of VNS treatment in a group of chronic and highly treatment-resistant patients compares favorably to pharmacotherapy outcomes demonstrated in STAR*D among a group of less severely ill patients. Indeed, VNS would be indicated for the type of patients that remain depressed after four treatment trials, such as those patients who completed treatment in STAR*D and were still depressed.

\section{Future Directions for Studying VNS}

Left cervical VNS was investigated and approved for the treatment of epilepsy and TRD, and right cervical VNS was avoided to minimize potential adverse cardiac effects. Animal and human studies, however, suggest that right cervical VNS is safe and effective for treating heart failure and arrhythmias [13••]. Left cervical VNS also has been shown to suppress atrial tachyarrhythmias in dogs [87], although VNS might be proarrhythmic under certain conditions [88]. Whether right, left, and bilateral cervical VNS have comparable efficacy, tolerability, and safety in the treatment of epilepsy or depression in humans is unknown. Krahl [3•] has suggested that there might be a lateralization of VNS effects (at least for seizure control). If so, would the modest antidepressant effects of left cervical VNS improve with the use of right or bilateral VNS? In depressed patients, it is also not known whether the potential benefits of VNS on cardiovascular, cerebrovascular, and metabolic risk factors would differ for right, left, or bilateral VNS. Addressing these clinically relevant issues in a clinical trial(s) is unlikely to occur. The design of such a study using a surgically implanted device with long-term assessments of relevant outcomes would be technically challenging and prohibitively expensive.

Transcutaneous VNS is a potentially viable option for conducting these types of studies, although its antidepressant efficacy has not been well studied and is not yet established. Right auricular t-VNS was found to inhibit atrial fibrillation in anesthetized dogs [89] and t-VNS altered proinflammatory biomarkers in a murine sepsis study [70]. Kreuzer and colleagues [90] reported that auricular t-VNS had a trend effect to shorten the QRS complex in patients treated for tinnitus, but the effect of t-VNS on inflammatory, metabolic, and/or cardiovascular biomarkers has not been extensively investigated in humans. Transcutaneous forms of VNS therefore deserve further study not only as a treatment for depression and other neuropsychiatric disorders, but also for its potentially favorable effects on cardiovascular, cerebrovascular, metabolic, and other physiological biomarkers that are associated with morbidity and mortality [68]. Using t-VNS would also permit an investigation of the relative clinical and neurobiological effects of right, left, and bilateral VNS.

\section{Compliance with Ethics Guidelines}

Conflict of Interest Robert H. Howland received a grant from Cyberonics, NeoSync, and Medtronic.

Human and Animal Rights and Informed Consent This article does not contain any studies with human or animal subjects performed by any of the authors.

\section{References}

Papers of particular interest, published recently, have been highlighted as:

- Of importance

•- Of major importance

1.• Ruffoli R, Giorgi FS, Pizzanelli C, et al. The chemical neuroanatomy of vagus nerve stimulation. J Chem Neuroanat. 2011;42:28896. This paper provides an overview of the neuroanatomy and neurochemistry relevant to the therapeutic uses of vagus nerve stimulation.

2. Zagon A. Does the vagus nerve mediate the sixth sense? Trends Neurosci. 2001;24(11):671-3.

3. Krahl SE. Vagus nerve stimulation for epilepsy: a review of the peripheral mechanisms. Surg Neurol Int. 2012;3(Suppl S1):47-52. This paper reviews the peripheral neuroanatomy and physiology of the vagus nerve that is relevant to the antiseizure effects of vagus nerve stimulation.

4. Lanska DJ. Corning and vagal nerve stimulation for seizures in the 1880s. Neurology. 2002;58:452-9.

5. Zabara J. Inhibition of experimental seizures in canines by repetitive vagal stimulation. Epilepsia. 1992;33(6):1005-12. 
6. Stancak A, Kuna M, Srinivasan, et al. KapalabhatiL yogic cleansing exercise. II. EEG topography analysis. Homeost Health Dis. 1991;3(4):182-9.

7. Stancak A, Pfeffer D, Hrudova L, et al. Electroencephalographic correlates of paced breathing. Neuroreport. 1993;4(6):723-6.

8. Morris GL, Gloss D, Buchhalter J, et al. Evidence-based guideline update: vagus nerve stimulation for the treatment of epilepsy. Neurology. 2013;81:1453-9. This is the most recent critical evaluation of evidence regarding the efficacy and safety of vagus nerve stimulation for epilepsy in children, adolescents, and adults.

9. Howland RH, Shutt LS, Berman SR, et al. The emerging use of technology for the treatment of depression and other neuropsychiatric disorders. Ann Clin Psychiatry. 2011;23:48-62.

10. Terry RS, Tarver WB, Zabara J. The implantable neurocybernetic prosthesis system. Pacing Clin Electrophysiol. 1991;14(1):86-93.

11. Gorny KR, Bernstein MA, Watson RE. 3 Tesla MRI of patients with vagus nerve stimulator: initial experience using a T/R head coil under controlled conditions. J Magn Reson Imaging. 2010;31:475-81.

12.• De Ferrari GM, Schwartz PJ. Vagus nerve stimulation: from preclinical to clinical application: challenges and future directions. Heart Fail Rev. 2011;16:195-203. This paper describes the preclinical and clinical background relevant to the therapeutic use of right cervical vagus nerve stimulation for heart failure.

13.• De Ferrari GM, Crijns HJGM, Borggrefe M, et al. Chronic vagus nerve stimulation: a new and promising therapeutic approach for chronic heart failure. Eur Heart J. 2011;32:847-55. This paper describes the results from the first phase II clinical trial of right cervical vagus nerve stimulation for heart failure.

14. Ben-Menachem E, Rydenhag B, Silander H. Preliminary experience with a new system for vagus nerve stimulation for the treatment of refractory focal onset seizures. Epilepsy Behav. 2013;29:416-9.

15.• Ellrich J. Transcutaneous vagus nerve stimulation. Eur Neurol Rev. 2011;6(4):254-6. This paper briefly describes the neuroanatomy of the auricular branch of the vagus nerve and the rationale for a transcutaneous form of vagus nerve stimulation.

16. Dietrich S, Smith J, Scherzinger C, et al. A novel transcutaneous vagus nerve stimulation leads to brainstem and cerebral activations measured by functional MRI. Biomed Tech (Berlin). 2008;53:104-11.

17. Kraus T, Hosl K, Kiess O, et al. BOLD fMRI deactivation of limbic and temporal brain structures and mood enhancing effect by transcutaneous vagus nerve stimulation. J Neural Transm. 2007;114:1485-93.

18.• Kraus T, Kiess O, Hosl K, et al. CNS BOLD fMRI effects of shamcontrolled transcutaneous electrical nerve stimulation in the left outer auditory canal: a pilot study. Brain Stimul. 2013;6:798-804. This paper describes the findings from an fMRI imaging study of the effects of transcutaneous stimulation of the outer auditory canal, demonstrating changes in limbic areas.

19. Ventureyra EC. Transcutaneous vagus nerve stimulation for partial onset seizure therapy. A new concept. Childs Nerv Syst. 2000;16: $101-2$.

20. Busch V, Zeman F, Heckel A, et al. The effect of transcutaneous vagus nerve stimulation on pain perception: an experimental study. Brain Stimul. 2013;6:202-9.

21. Lehtimaki J, Hyvarinen P, Ylikoski M, et al. Transcutaneous vagus nerve stimulation in tinnitus: a pilot study. Acta Otolaryngol. 2013;133:378-82.

22.• Stefan H, Kreiselmeyer G, Kerling F, et al. Transcutaneous vagus nerve stimulation (t-VNS) in pharmacoresistant epilepsies: a proof of concept trial. Epilepsia. 2012;53(7):e115-8. This paper describes the findings from a pilot study of transcutaneous auricular vagus nerve stimulation in adults with refractory epilepsy.

23. He W, Jing X, Wang X, et al. Transcutaneous auricular vagus nerve stimulation as a complementary therapy for pediatric epilepsy: a pilot trial. Epilepsy Behav. 2013;28:343-6. This paper describes the results of a pilot study of transcutaneous auricular vagus nerve stimulation for epilepsy in children.
24. Peijing R, Aihua L, Jianguo Z, et al. An alternative therapy for drugresistant epilepsy: transcutaneous auricular vagus nerve stimulation. Chin Med J. 2014;127(2):300-4.

25.• Hein E, Nowak M, Kiess O, et al. Auricular transcutaneous electrical nerve stimulation in depressed patients: a randomized controlled pilot study. J Neural Transm. 2013;120:821-7. This paper describes the findings from a pilot study of transcutaneous vagus nerve stimulation for non-chronic non-refractory major depression.

26. Hassanzadeh R, Jone JC, Ross EL. Neuromodulation for intractable headaches. Curr Pain Headache Rep. 2014;18:392. doi:10.1007/ s11916-013-0392-1.

27. George MS, Rush AJ, Sackeim HA, Marangell LB. Vagus nerve stimulation (VNS): utility in neuropsychiatric disorders. Int $\mathrm{J}$ Neuropsychopharmacol. 2003;6:73-83.

28. Krahl SE, Senanayake SS, Pekary AE, Sattin A. Vagus nerve stimulation (VNS) is effective in a rat model of antidepressant action. J Psychiatr Res. 2004;38:237-40.

29. Furmaga H, Shah A, Frazer A. Serotonergic and noradrenergic pathways are required for the anxiolytic-like and antidepressantlike behavioral effects of repeated vagal nerve stimulation in rats. Biol Psychiatry. 2011;70:937-45.

30. Kosel M, Brockmann H, Frick C, et al. Chronic vagus nerve stimulation for treatment-resistant depression increases regional cerebral blood flow in the dorsolateral prefrontal cortex. Psychiatry Res. 2011;191:153-9. A recent brain imaging study of the effects of invasive left cervical vagus nerve stimulation in patients with treatment resistant depression.

31. Conway CR, Chibnall JT, Gebarea MA, et al. Association of cerebral metabolic activity changes with vagus nerve stimulation antidepressant response in treatment-resistant depression. Brain Stimul. 2013;6:788-97. A recent brain imaging study investigating correlations between cerebral metabolic activity and the treatment response to left cervical vagus nerve stimulation.

32. Furmaga H, Carreno FR, Frazer A. Vagal nerve stimulation rapidly activates brain-derived neurotrophic factor receptor TrkB in rat brain. PLoS ONE. 2012;7(5):e34844. doi:10.1371/journal.pone. 0034884.

33. Gebhardt N, Bar KJ, Boettger MK, et al. Vagus nerve stimulation ameliorated deficits in one-way active avoidance learning and stimulated hippocampal neurogenesis in bulbectomized rats. Brain Stimul. 2013;6:78-83.

34. Rush AJ, George MS, Sackeim HA, et al. Vagus nerve stimulation (VNS) for treatment-resistant depressions: a multicenter study. Biol Psychiatry. 2000;47:276-86.

35. Sackeim HA, Rush AJ, George MS, et al. Vagus nerve stimulation (VNS) for treatment-resistant depression: efficacy, side effects, and predictors of outcome. Neuropsychopharmacology. 2001;25:71328.

36. Marangell LB, Rush AJ, George MS, et al. Vagus nerve stimulation (VNS) for major depressive episodes: one year outcomes. Biol Psychiatry. 2002;51:280-7.

37. Nahas Z, Marangell LB, Husain MM, et al. Two-year outcome of vagus nerve stimulation (VNS) for treatment of major depressive episodes. J Clin Psychiatry. 2005;66:1097-104.

38. Dunner DL, Rush AJ, Russell JM, et al. Prospective long-term multicenter study of the naturalistic outcomes of patients with treatment-resistant depression. J Clin Psychiatry. 2006;67:688-95.

39. Rush AJ, Marangell LB, Sackeim HA, et al. Vagus nerve stimulation for treatment-resistant depression: a randomized controlled acute phase trial. Biol Psychiatry. 2005;58:347-54.

40. Rush AJ, Sackeim HA, Marangell LB, et al. Effects of 12 months of vagus nerve stimulation in treatment-resistant depression: a naturalistic study. Biol Psychiatry. 2005;58:355-63.

41. George MS, Rush AJ, Marangell LB, et al. A one-year comparison of vagus nerve stimulation with treatment as usual for treatmentresistant depression. Biol Psychiatry. 2005;58:364-73. 
42. Nierenberg AA, Alpert JE, Gardner-Schuster EE, et al. Vagus nerve stimulation: 2-year outcomes for bipolar versus unipolar treatmentresistant depression. Biol Psychiatry. 2008;64(6):455-60.

43. Schlaepfer TE, Frick C, Zobel A, et al. Vagus nerve stimulation for depression: efficacy and safety in a European study. Psychol Med. 2008;38:651-61.

44. Bajbouj M, Merkl A, Schlaepfer TE, et al. Two-year outcome of vagus nerve stimulation in treatment-resistant depression. J Clin Psychopharmacol. 2010;30:273-81.

45. Aaronson ST, Carpenter LL, Conway CR, et al. Vagus nerve stimulation therapy randomized to different amounts of electrical charge for treatment-resistant depression: acute and chronic effects. Brain Stimul. 2013;6:631-40. This paper describes the results of a recent large randomized clinical trial comparing outcomes for three different stimulation doses of left cervical vagus nerve stimulation in patients with treatment resistant depression.

46. Muller HH, Kornhuber J, Maler JM, Sperling W. The effects of stimulation parameters on clinical outcomes in patients with vagus nerve stimulation implants with major depression. J ECT. 2013;29: e40-2.

47.• Berry SM, Broglio K, Bunker M, et al. A patient-level metaanalysis of studies evaluating vagus nerve stimulation therapy for treatment-resistant depression. Med Devices (Auckl). 2013;6:1735. This paper describes the findings from an analysis of patientlevel data from six large prospective studies of left cervical vagus nerve stimulation compared with treatment-as-usual for treatment resistant depression.

48. Olin B, Jayewardene AK, Bunker M, Moreno F. Mortality and suicide risk in treatment-resistant depression: an observational study of the long-term impact of intervention. PLoS ONE. 2012;7(10): e48002. doi:10.1371/journal.pone.0048002. This paper describes the initial findings from a vagus nerve stimulation therapy registry of patients with treatment resistant depression.

49. Gerson R, Murray E, Price B, et al. Mania following vagus nerve stimulation: a case report and review of the literature. Epilepsy Behav. 2011;20:138-40. This paper provides a brief literature review of the risk of mania associated with vagus nerve stimulation.

50. Feldman RL, Dunner DL, Muller JS, Stone DA. Medicare patient experience with vagus nerve stimulation for treatment-resistant depression. J Med Econ. 2013;16(1):62-74. doi:10.3111/ 136969987.2012.724745.

51. Rong PJ, Fang JL, Wang LP, et al. Transcutaneous vagus nerve stimulation for the treatment of depression: a study protocol for a double blinded randomized clinical trial. BMC Complement Alternat Med. 2012;12:255. doi:10.1186/1472-6882-12-255.

52. Rugulies R. Depression as a predictor for coronary heart disease: a review and meta-analysis. Am J Prev Med. 2002;23:51-61.

53. Carney RM, Freedland KE, Miller GE, Jaffe AS. Depression as a risk factor for cardiac mortality and morbidity: a review of potential mechanisms. J Psychosom Res. 2002;53:897-902.

54. Brown AD, Barton DA, Lambert GW. Cardiovascular abnormalities in patients with major depressive disorder: autonomic mechanisms and implications for treatment. CNS Drugs. 2009;23(7):583602.

55. Li W, Olshansky B. Inflammatory cytokines and nitric oxide in heart failure and potential modulation by vagus nerve stimulation. Heart Fail Rev. 2011;16:137-45.

56. Abboud FM, Harwani SC, Chapleau MW. Autonomic neural regulation of the immune system: implications for hypertension and cardiovascular disease. Hypertension. 2012;59:755-62.

57. Huston JM, Tracey KJ. The pulse of inflammation: heart rate variability, the cholinergic anti-inflammatory pathway and implications for therapy. J Intern Med. 2011;269:45-53.

58. Anders S, Tanaka M, Kinney DK. Depression as an evolutionary strategy for defense against infection. Brain Behav Immun. 2013;31:9-22.
59. Tanaka M, Anders S, Kinney DK. Environment, the immune system, and depression: an integrative review and discussion of the infection-defense hypothesis. In: Dietert RR, Luebke RW, editors. Immunotoxicity, immune dysfunction, and chronic disease. Humana Press (Springer), New York. 2012; pp 345-85.

60. Benros ME, Waltoft BL, Nordentoft M, et al. Autoimmune diseases and severe infections as risk factors for mood disorders: a nationwide study. JAMA Psychiatry. 2013;70:812-20.

61. Capuron L, Miller AH. Immune system to brain signaling: neuropsychopharmacological implications. Pharmacol Ther. 2011;130:226-38.

62. Hornig M. The role of microbes and autoimmunity in the pathogenesis of neuropsychiatric illness. Curr Opin Rheumatol. 2013;25(4):488-95.

63. Cryan JF, Dinan TG. Mind-altering microorganisms: the impact of the gut microbiota on brain and behavior. Nat Rev Neurosci. 2012;13:701-12.

64. Raison CL, Lowry CA, Rook GAW. Inflammation, sanitation, and consternation: loss of contact with coevolved, tolerogenic microorganisms and the pathophysiology and treatment of major depression. Arch Gen Psychiatry. 2010;67:1211-24.

65. Hannestad J, DellaGioia N, Bloch M. The effect of antidepressant medication treatment on serum levels of inflammatory cytokines: a meta-analysis. Neuropsychopharmacology. 2011;36:2452-9.

$66 . •$ Bonaz B, Picq C, Sinniger V, et al. Vagus nerve stimulation: from epilepsy to the cholinergic anti-inflammatory pathway. Neurogastroenterol Motil. 2013;25:208-21. This paper reviews the involvement of the vagus nerve in the cholinergic antiinflammatory pathway and the known and potential antiinflammatory effects of vagus nerve stimulation.

67.• Pavlov VA, Tracey KJ. The vagus nerve and the inflammatory reflex: linking immunity and metabolism. Nat Rev Endocrinol. 2012;8:743-54. This paper reviews the involvement of the vagus nerve in regulation of metabolic homeostasis, and the efferent vagus nerve-mediated control of immune function and proinflammatory response via the cholinergic inflammatory reflex.

68. Sun Z, Baker W, Hiraki T, Greenberg JH. The effect of right vagus nerve stimulation on focal cerebral ischemia. Brain Stimul. 2012;5: $1-10$.

69. Ay I, Sorensen AG, Ay H. Vagus nerve stimulation reduces infarct size in rat focal cerebral ischemia: an unlikely role for cerebral blood flow. Brain Res. 2011;1392:110-5.

70. Huston JM, Gallowitsche-Puerta M, Ochani M, et al. Transcutaneous vagus nerve stimulation reduces serum high mobility group box 1 levels and improves survival in murine sepsis. Crit Care Med. 2007;35:2762-8.

71. Aalbers MW, Klinkenberg S, Rijkers K, et al. The effects of vagus nerve stimulation on pro- and anti-inflammatory cytokines in children with refractory epilepsy: an exploratory study. Neuroimmunomodulation. 2012;19:352-8.

72. Barone L, Colicchio G, Policicchio D, et al. Effect of vagal nerve stimulation on systemic inflammation and cardiac autonomic function in patients with refractory epilepsy. Neuroimmunomodulation. 2007;14:331-6.

73. Majoie HJ, Rijkers K, Berfelo MW, et al. Vagus nerve stimulation in refractory epilepsy: effects on pro- and anti-inflammatory cytokines in peripheral blood. Neuroimmunomodulation. 2011;18:52-6.

74. De Herdt V, Bogaert S, Bracke KR, et al. Effects of vagus nerve stimulation on pro- and anti-inflammatory cytokine induction in patients with refractory epilepsy. J Neuroimmunol. 2009;214: $104-8$.

75. Corcoran C, Connor TJ, O'Keane V, Garland MR. The effects of vagus nerve stimulation on pro- and anti-inflammatory cytokines in humans: a preliminary report. Neuroimmunomodulation. 2005;12: 307-9. 
76. Moreno J, Gaspar E, Lopez-Bello G, et al. Increase in nitric oxide levels and mitochondrial membrane potential in platelets of untreated patients with major depression. Psychiatry Res. 2013;209:447-52.

77. Trueba AF, Smith NB, Auchus RJ, Ritz T. Academic exam stress and depressive mood are associated with reductions in exhaled nitric oxide in healthy individuals. Biol Psychol. 2013;93:206-12.

78. Lu DY, Leung YM, Su KP. Interferon-alpha induces nitric oxide synthase expression and haem oxygenase-1 down-regulation in microglia: implications of cellular mechanism of IFN-alphainduced depression. Int J Neuropsychopharmacol. 2013;16:43344.

79.• Leonard BE. Impact of inflammation on neurotransmitter changes in major depression: an insight into the action of antidepressants. Prog Neuro-Psychopharmacol Biol Psychiatry. 2014;48:261-7. This review summarizes evidence that chronic low-grade inflammation plays an important role in the pathology of depression.

80. Lotrich F. Inflammatory cytokines, growth factors, and depression. Curr Pharm Des. 2012;18:5920-35.

81. Raison CL, Rutherford RE, Woolwine BJ, et al. A randomized controlled trial of the tumor necrosis factor antagonist infliximab for treatment-resistant depression: the role of baseline inflammatory biomarkers. JAMA Psychiatry. 2013;70:31-41.

82. Celano CM, Huffman JC. Depression and cardiac disease: a review. Cardiol Rev. 2011;19:130-42.

83. Rustad JK, Stern TA, Hebert KA, Musselman DL. Diagnosis and treatment of depression in patients with congestive heart failure: a review of the literature. Prim Care Companion CNS Disord. 2013;15, PCC.13r01511. doi:10.4088/PCC.13r01511.

84. Martin JLR, Martin-Sanchez E. Systematic review and metaanalysis of vagus nerve stimulation in the treatment of depression: variable results based on study design. Eur Psychiatry. 2012;27: 147-55.

85. Olanchanski N, McInnis Myers M, Halseth M, et al. The economic burden of treatment-resistant depression. Clin Ther. 2013;35:51222.

86. Rush AJ, Trivedi MH, Wisniewski SR, et al. Acute and longer-term outcomes in depressed outpatients requiring one or several treatment steps: a STAR*D report. Am J Psychiatry. 2006;163:1905-17.

87. Shen MJ, Shinohara T, Park HW, et al. Continuous low-level vagus nerve stimulation reduces stellate ganglion nerve activity and paroxysmal atrial tachyarrhythmias in ambulatory canines. Circulation. 2011;123:2204-12.

88. Zhang Y, Mazgalev TN. Arrhythmias and vagus nerve stimulation. Heart Fail Rev. 2011;16:147-61.

89. Yu L, Scherlag BJ, Li S, et al. Low-level transcutaneous electrical stimulation of the auricular branch of the vagus nerve: a noninvasive approach to treat the initial phase of atrial fibrillation. Heart Rhythm. 2013;10:428-35.

90. Kreuzer PM, Landgrebe J, Husser O, et al. Transcutaneous vagus nerve stimulation: retrospective assessment of cardiac safety in a pilot study. Front Psychiatry. 2012;3:70. doi:10.3389/fpsyt.2012. 00070 . 